\title{
Rentabilidade da cultura da macieira cultivar Gala em duas densidades de plantio(1)
}

\begin{abstract}
Carlos Leomar Kreuz ${ }^{(2)}$
Resumo - O presente trabalho discute, com base em um estudo de caso, a viabilidade econômica de ampliar a densidade de plantio de macieiras cultivar Gala de 1.000 plantas por hectare para 3.378 plantas por hectare. Os resultados mostraram que a taxa interna de retorno não variou (próxima de $1,61 \%$ ao mês), independentemente da densidade utilizada, o que sugere o uso da menor densidade de plantio, uma vez que esta requer um menor volume de recursos financeiros. O custo unitário de produção também se mantém praticamente o mesmo ao se variar a densidade de plantio.
\end{abstract}

Termos para indexação: Malus domestica, fruteiras, espaçamento, taxa de crescimento, modelos matemáticos.

\section{Investment return for Gala apple cultivar using two planting densities}

Abstract - The present study was carried out to analyse economic performances of Gala apple orchards at different planting densities. Two orchard densities ( 1,000 and 3,378 plants/ha) were evaluated in an orchard near Fraiburgo, SC, Brazil. The use of both planting densities resulted in the same internal rate of return (approximately 1.6\% a month). It is suggested to use a lower planting system, once it requires less capital. Unit cost also remained the same for both planting densities used in this study.

Index terms: Malus domestica, fruit crops, spacing, growth rate, mathematical models.

\section{Introdução}

Na cultura da macieira, por se tratar de uma cultura perene, a decisão sobre qual seja o espaçamento entre plantas e entre filas a utilizar por ocasião do plantio repercutirá durante toda a vida do pomar. As implicações da densidade de plantio escolhida são as mais variadas, e interferem, entre outros aspectos, na coloração dos frutos (Wertheim et al., 1986; Petri et al., 1991), na produtividade (Wertheim et al., 1986; Sansavini, 1998), na qualidade da produção (Robinson et al., 1991a), no peso médio dos frutos (Robinson et al., 1991a), na interceptação da luz (Robinson \& Lakso, 1991), no porte das plantas (Robinson et al., 1991b), e na necessidade de capital (Ferree et al., 1989).

Análises econômicas comparando diferentes densidades de plantas tendem a converter as variações

\footnotetext{
(1) Aceito para publicação em 19 de junho de 2001.

(2)Empresa de Pesquisa Agropecuária e Extensão Rural de Santa Catarina, Caixa Postal 591, CEP 89500-000 Caçador, SC.

E-mail: kreuz@epagri.rct-sc.br
}

produtivas e qualitativas em unidades monetárias, facilitando a decisão do produtor. Contudo, apesar da nítida preferência européia por pomares plantados em densidades maiores (Goedegebure, 1980; Winter, 1986), ou de uma indicação para o uso de espaçamentos menores em pomares da Nova Zelândia (Cahn-Shaharudin, 1991; Palmer \& Adams, 1997), Jackson et al. (1986), Ferree et al. (1989) e Dejong et al. (1999) concluem que o uso de alta densidade pode não ser interessante do ponto de vista econômico, uma vez que os maiores custos iniciais dos pomares em alta densidade não são compensados pela antecipação obtida nas produções. Por outro lado, Robinson et al. (1996) mostram que a rentabilidade do pomar aumenta com o aumento da densidade de plantio até atingir um ponto de máximo, e decai a partir deste ponto.

Na região brasileira produtora de maçãs, a dúvida quanto à densidade ideal persiste. Enquanto a região de São Joaquim, SC, tem optado por densidades menores, as de Vacaria, RS, e Fraiburgo, SC, tendem a preferir densidades maiores. Kreuz et al. (1996), estudando a cultivar Fuji enxertada sobre os porta- 
enxertos MM-106 e M-7, recomendam, com base na evolução da capacidade produtiva das plantas, o uso de 4,5 m entre filas e 1,25 a 1,50 m entre plantas. Contudo, não são conhecidas avaliações econômicas envolvendo pomares em alta densidade nas condições do sul do Brasil.

O objetivo deste trabalho foi verificar a rentabilidade de duas densidades de plantas usadas nos pomares comerciais existentes na região de Fraiburgo, SC.

\section{Material e Métodos}

As análises foram realizadas tomando-se como base os dados provenientes de uma empresa comercial instalada no Município de Fraiburgo, SC. A condição edafoclimática do local encontra-se descrita em Petri (1986).

Os coeficientes técnicos foram coletados de planilhas da empresa, onde todas as atividades nos pomares haviam sido registradas. Os preços dos insumos utilizados tiveram por base os valores praticados na última compra realizada pela empresa em outubro de 2000.

Foram utilizados os dados de densidades de plantio de 1.000 plantas por hectare (espaçamento de $5,0 \mathrm{~m} \mathrm{x} \mathrm{2,0} \mathrm{m)}$ e 3.378 plantas por hectare $(3,7 \mathrm{~m} \times 0,80 \mathrm{~m})$, da macieira cultivar Gala enxertada sobre o porta-enxerto MM-106 e M-9, respectivamente, no sistema de "líder central". O manejo dos pomares seguiu as recomendações da pesquisa agropecuária (Empresa de Pesquisa Agropecuária de Santa Catarina, 1991; Barrit, 1999). Os custos de implantação entre essas duas densidades diferiram, basicamente, no custo das mudas ( $\mathrm{R} \$ 1,80 /$ muda) e no custo de tutoramento ( $\mathrm{R} \$ 1.500,00 / \mathrm{ha}$ ) necessário para a maior densidade de plantio. Por ocasião das fases iniciais dos pomares, a principal diferença de custos se deu na colheita ( $\mathrm{R} \$ 27,90 / \mathrm{t})$, sendo este maior na alta densidade, em face do maior volume colhido. Com relação ao pomar adulto, a maior densidade de plantio possuía vantagens em termos do uso de mão-de-obra, e este foi reduzido em $25 \%$ em comparação ao pomar menos denso. Isto se deve ao fato de o menor porte das plantas facilitar as operações manuais.

As informações relativas à produtividade nos diferentes anos após o plantio tiveram por base os resultados atingidos pela empresa nos pomares em produção. Basicamente, nas densidades maiores as produtividades iniciais foram maiores. Contudo, à medida que atingiam a idade adulta, a produtividade de pomares em alta e baixa densidade de plantio foi praticamente a mesma. Isto é possível pelo fato de se tratar do mesmo sistema de condução (líder central), da mesma cultivar (Gala), e em virtude do ajuste no porta-enxerto (em 1.000 plantas/ha se usa o MM-106 e em 3.378 plantas/ha se usa o M-9).

O modelo matemático de análise usado foi o do cálculo da Taxa Interna de Retorno (TIR) (Vieira Sobrinho, 1997):

$\mathrm{FC}_{0}-\sum_{\mathrm{j}=1}^{\mathrm{n}} \mathrm{FC}_{\mathrm{j}} /(1+\mathrm{i})^{\mathrm{j}}=0$

onde: $\mathrm{FC}_{0}$ representa o fluxo de caixa inicial (ano zero do investimento); $\mathrm{FC}_{\mathrm{j}}$ representa o fluxo de caixa no j-ésimo ano do empreendimento; $\mathrm{n}$ representa o último ano do empreendimento (derrubada do pomar); i representa a taxa interna de retorno. Para fins operacionais, todos os desembolsos (fluxo de caixa negativo) são representados com o sinal negativo, e todas as receitas (fluxo de caixa positivo) o são com o sinal positivo. O fluxo de caixa é obtido pela subtração entre as receitas e os custos do ano, supondo que os mesmos ocorram, para facilitar o cálculo, simultaneamente no início do período.

As receitas são obtidas pelo produto entre a produção estimada no ano pelo preço de venda. Este tem por base o valor de venda de maçã antes da classificação, armazenagem e embalagem, sendo de $\mathrm{R} \$ 0,25 / \mathrm{kg}$. Porém, supõe-se que $10 \%$ da produção seja de qualidade industrial, sem valor comercial. Desta forma, o preço médio de venda se reduz para $\mathrm{R} \$ 0,225 / \mathrm{kg}$, o qual sofre, ainda, interferência da densidade ( $\mathrm{R} \$ 0,225 / \mathrm{kg}$ com relação à densidade de 1.000 plantas e $\mathrm{R} \$ 0,236 / \mathrm{kg}$ com relação à densidade de 3.378 plantas). Usou-se um preço de venda acrescido em $5 \%$ para a maçã proveniente do pomar de maior densidade, em razão da melhor coloração dos frutos, o que está de acordo com Petri et al. (1991).

$\mathrm{Na}$ determinação do custo do quilograma produzido em cada densidade de plantio, simulou-se em (1) preços de venda que levem à mínima TIR aceitável do ponto de vista de investimento, ou seja, $0,5 \%$ ao mês. Este método difere do usado por Kreuz (1992), no qual se utilizou, para a obtenção do custo do quilo, o cociente entre o valor descontado dos custos e das produções estimadas.

Tendo-se em vista o modelo de análise de investimentos proposto (1), há necessidade de se acrescentar o valor da terra por ocasião da instalação do pomar (apesar de alguns autores, como Jackson et al. (1986), não a considerarem). Por outro lado, a terra se caracterizará como uma receita por ocasião do término da vida útil do pomar. O custo da erradicação do pomar não se faz necessário, uma vez que os coeficientes técnicos a serem considerados no custo da instalação do pomar já contemplam esta necessidade. Estima-se como sendo de 16 anos a vida útil do pomar, considerada razoável para os tempos atuais em que o constante lançamento de cultivares reduz o seu ciclo de mercado. 


\section{Resultados e Discussão}

Na Tabela 1 é apresentada a necessidade de capital para a implantação de 1 ha de macieiras em uma densidade de 1.000 plantas/ha. Supondo-se como sendo de R $\$ 1.500,00$ o valor da terra, o montante do investimento inicial atingiria $\mathrm{R} \$ 6.423,00$. $\mathrm{Na}$ densidade de 3.378 plantas/ha, o montante do investimento inicial chega em R \$ 12.203,40, uma vez que há necessidade de se acrescentar o custo do tutoramento ( $\mathrm{R} \$ 1.500,00)$ e as mudas adicionais ( $\mathrm{R} \$ 4.280,40)$. O custo das mudas é o maior custo individual na instalação do pomar, aproximando-se do próprio valor da terra para o caso de uma densidade de plantio de 1.000 mudas/ha.

$\mathrm{O}$ custo de manutenção do pomar enquanto jovem (primeiros três anos) é crescente com a idade
(Tabela 2). No primeiro ano, o custo de manutenção não difere entre as densidades estudadas. No segundo e no terceiro ano, os custos de manutenção diferem entre as duas densidades. Na densidade de 3.378 plantas/ha, no segundo ano, este é acrescido do custo da primeira colheita $(\mathrm{R} \$ 139,50)$. No terceiro ano, dado que a maior densidade possibilita um maior volume de colheita, o custo de manutenção passa a ser de $\mathrm{R} \$ 3.925,10$.

Um pomar adulto, produzindo $50 \mathrm{t} / \mathrm{ha}$, em uma densidade de 1.000 plantas/ha (Tabela 3), requer $\mathrm{R} \$ 5.043,63$, o qual pode ser decomposto em insumos, serviços de máquinas e serviços manuais e outras despesas. Desta forma, os insumos correspondem a $42 \%$ do custo anual, seguido dos serviços manuais (36\%), serviços mecanizados (12\%) e outras despesas (11\%). O custo anual de um po-

Tabela 1. Custo de instalação de 1 ha de pomar de macieira cultivar Gala na densidade de 1.000 plantas/ha, em outubro de 2000 .

\begin{tabular}{|c|c|c|c|c|}
\hline Item & Unidade $^{(1)}$ & Quantidade & $\mathrm{R} \$$ /unidade & $\mathrm{R} \$ / \mathrm{ha}$ \\
\hline \multicolumn{5}{|l|}{ Preparo do solo } \\
\hline Subsolagem tripla & $\mathrm{h} / \operatorname{tr} \mathrm{E}$ & 6 & 40,00 & 240,00 \\
\hline Limpeza de raízes & $\mathrm{d} / \mathrm{h}$ & 5 & 9,00 & 45,00 \\
\hline Limpeza de pedras & $\mathrm{h} / \mathrm{tr}$ & 8 & 13,50 & 108,00 \\
\hline Limpeza de pedras & $\mathrm{d} / \mathrm{h}$ & 12 & 9,00 & 108,00 \\
\hline Sub-total & & & & 501,00 \\
\hline \multicolumn{5}{|l|}{ Correção do solo } \\
\hline Calcário & $\mathrm{t}$ & 25 & 22,00 & 550,00 \\
\hline Hiperfosfato & $\mathrm{kg}$ & 2.500 & 0,18 & 450,00 \\
\hline Cloreto de potássio & $\mathrm{kg}$ & 300 & 0,22 & 66,00 \\
\hline Bórax & $\mathrm{kg}$ & 50 & 1,00 & 50,00 \\
\hline Aplicação de calcário e adubos & $\mathrm{h} / \mathrm{tr}$ & 12 & 13,50 & 162,00 \\
\hline Sub-total & & & & $1.278,00$ \\
\hline \multicolumn{5}{|l|}{ Preparo do solo pós-correção } \\
\hline Lavração & $\mathrm{h} / \mathrm{tr}$ & 6 & 13,50 & 81,00 \\
\hline Limpezas & $\mathrm{d} / \mathrm{h}$ & 8 & 9,00 & 72,00 \\
\hline Gradagem & $\mathrm{h} / \mathrm{tr}$ & 4 & 13,50 & 54,00 \\
\hline Sub-total & & & & 207,00 \\
\hline \multicolumn{5}{|l|}{ Plantio } \\
\hline Marcação & $\mathrm{d} / \mathrm{h}$ & 5 & 9,00 & 45,00 \\
\hline Abert. de covas e plantio & $d / h$ & 30 & 9,00 & 270,00 \\
\hline Abertura de covas & $\mathrm{h} / \mathrm{tr}$ & 12 & 13,50 & 162,00 \\
\hline Mudas (Espaç.5,0x2,0 m) & unid. & 1.000 & 1,80 & $1.800,00$ \\
\hline Mão-de-obra & $\mathrm{d} / \mathrm{h}$ & 40 & 9,00 & 360,00 \\
\hline Materiais diversos & & & & 300,00 \\
\hline Sub-total & & & & $2.937,00$ \\
\hline Valor da terra & $\mathrm{R} \$ /$ ha & & & $1.500,00$ \\
\hline Total & & & & $6.423,00$ \\
\hline
\end{tabular}

${ }^{(1)} \mathrm{h} /$ tr E: hora de trator de esteira; $\mathrm{d} / \mathrm{h}$ : dias homem; h/tr: horas trator; unid.: unidade. 
Tabela 2. Custo de condução de 1 ha de pomar de macieira cultivar Gala com 1.000 plantas/ha, nos 3 anos após a instalação, em outubro de 2000.

\begin{tabular}{|c|c|c|c|c|c|c|c|c|}
\hline \multirow[t]{2}{*}{ Descrição } & \multirow[t]{2}{*}{ Unidade } & \multirow{2}{*}{$\begin{array}{c}\text { Preço } \\
\mathrm{R} \$ / \text { unidade }\end{array}$} & \multicolumn{2}{|c|}{$1^{\circ}$ ano } & \multicolumn{2}{|c|}{$2^{\circ}$ ano } & \multicolumn{2}{|c|}{$3^{\circ}$ ano } \\
\hline & & & Quantidade & $\mathrm{R} \$$ & Quantidade & $\mathrm{R} \$$ & Quantidade & $\mathrm{R} \$$ \\
\hline \multicolumn{9}{|l|}{ Fertilizantes } \\
\hline Adubo $10-10-10$ & $\mathrm{~kg}$ & 0,35 & & & 200,00 & 70,00 & 250,00 & 87,50 \\
\hline Nitrocálcio & $\mathrm{kg}$ & 0,40 & 200,00 & 80,00 & 200,00 & 80,00 & 250,00 & 100,00 \\
\hline Cloreto de cálcio & $\mathrm{kg}$ & 0,48 & & & & 0,00 & 10,00 & 4,80 \\
\hline Sub-total & & & & 80,00 & & 150,00 & & 192,30 \\
\hline \multicolumn{9}{|l|}{ Fungicidas } \\
\hline Baycor & $\mathrm{kg}$ & 36,00 & 1,00 & 36,00 & 1,00 & 36,00 & 2,00 & 72,00 \\
\hline Benlate & $\mathrm{kg}$ & 24,00 & 0,50 & 12,00 & 1,00 & 24,00 & 1,50 & 36,00 \\
\hline Captan & $\mathrm{kg}$ & 4,40 & 5,00 & 22,00 & 8,00 & 35,20 & 10,00 & 44,00 \\
\hline Cercobin & $\mathrm{kg}$ & 11,60 & 1,00 & 11,60 & 1,50 & 17,40 & 2,00 & 23,20 \\
\hline Delan & $\mathrm{kg}$ & 30,00 & 0,50 & 15,00 & 1,00 & 30,00 & 1,50 & 45,00 \\
\hline Dithane & $\mathrm{kg}$ & 4,40 & 6,00 & 26,40 & 12,00 & 52,80 & 20,00 & 88,00 \\
\hline Recop & $\mathrm{kg}$ & 2,00 & 4,00 & 8,00 & 5,00 & 10,00 & 6,00 & 12,00 \\
\hline Rubigan & $\mathrm{L}$ & 43,00 & 0,30 & 12,90 & 0,50 & 21,50 & 0,80 & 34,40 \\
\hline Saprol & $\mathrm{L}$ & 14,00 & 0,50 & 7,00 & 1,00 & 14,00 & 1,50 & 21,00 \\
\hline Thiovit & $\mathrm{kg}$ & 1,40 & 2,50 & 3,50 & 5,00 & 7,00 & 10,00 & 14,00 \\
\hline Venturol & $\mathrm{kg}$ & 17,00 & 1,00 & 17,00 & 2,00 & 34,00 & 3,00 & 51,00 \\
\hline Outros & & & & 60,00 & & 120,00 & & 180,00 \\
\hline Sub-total & & & & 231,40 & & 401,90 & & 620,60 \\
\hline \multicolumn{9}{|l|}{ Inseticidas } \\
\hline Acaristop & $\mathrm{L}$ & 120,00 & & 0,00 & 0,40 & 48,00 & 0,50 & 60,00 \\
\hline Decis & $\mathrm{L}$ & 17,00 & 0,20 & 3,40 & 0,40 & 6,80 & 0,80 & 13,60 \\
\hline Formicida & $\mathrm{kg}$ & 2,00 & 3,00 & 6,00 & 3,00 & 6,00 & 3,00 & 6,00 \\
\hline Imidan & $\mathrm{L}$ & 9,20 & 2,00 & 18,40 & 3,00 & 27,60 & 4,00 & 36,80 \\
\hline Óleo triona & $\mathrm{L}$ & 0,80 & 5,00 & 4,00 & 25,00 & 20,00 & 35,00 & 28,00 \\
\hline Omite & $\mathrm{L}$ & 16,50 & & 0,00 & 2,00 & 33,00 & 2,00 & 33,00 \\
\hline Perfecthion & $\mathrm{L}$ & 4,80 & & 0,00 & 1,00 & 4,80 & 1,50 & 7,20 \\
\hline Sipcatin & $\mathrm{L}$ & 44,00 & 0,50 & 22,00 & 0,60 & 26,40 & 0,60 & 26,40 \\
\hline Outros & & & & 12,00 & & 36,00 & & 60,00 \\
\hline Sub-total & & & & 65,80 & & 208,60 & & 271,00 \\
\hline \multicolumn{9}{|l|}{ Herbicidas e outros } \\
\hline Roundup & $\mathrm{L}$ & 7,80 & & 0,00 & & 0,00 & 2,00 & 15,60 \\
\hline Gramoxone & $\mathrm{L}$ & 7,00 & & 0,00 & 0,50 & 3,50 & 1,00 & 7,00 \\
\hline Ag-bem & $\mathrm{L}$ & 4,40 & 2,00 & 8,80 & 4,00 & 17,60 & 6,00 & 26,40 \\
\hline Dormex & $\mathrm{L}$ & 11,20 & 0,50 & 5,60 & 2,50 & 28,00 & 3,50 & 39,20 \\
\hline Outros & & Dvs. & & 250,00 & & 375,00 & & 500,00 \\
\hline Sub-total & & & & 264,40 & & 424,10 & & 588,20 \\
\hline \multicolumn{9}{|l|}{ Servicos } \\
\hline Aplic. fertilizantes & $\mathrm{h} / \mathrm{tr}$ & 13,50 & 1,00 & 13,50 & 2,00 & 27,00 & 2,00 & 27,00 \\
\hline Aplic. fertilizantes & $\mathrm{d} / \mathrm{h}$ & 9,00 & 1,00 & 9,00 & 2,00 & 18,00 & 2,00 & 18,00 \\
\hline Cultivos mecânicos & $\mathrm{h} / \mathrm{tr}$ & 13,50 & 3,00 & 40,50 & 5,00 & 67,50 & 5,00 & 67,50 \\
\hline Cultivos manuais & $\mathrm{d} / \mathrm{h}$ & 9,00 & 10,00 & 90,00 & 10,00 & 90,00 & 15,00 & 135,00 \\
\hline Podas / conduções & $\mathrm{d} / \mathrm{h}$ & 9,00 & 8,00 & 72,00 & 10,00 & 90,00 & 12,00 & 108,00 \\
\hline Transporte de insumos & $\mathrm{h} / \mathrm{tr}$ & 13,50 & 2,00 & 27,00 & 4,00 & 54,00 & 4,00 & 54,00 \\
\hline Conduções diversas & $\mathrm{d} / \mathrm{h}$ & 9,00 & 5,00 & 45,00 & 10,00 & 90,00 & 15,00 & 135,00 \\
\hline Aplic. de defensivos & $\mathrm{h} / \mathrm{tr}$ & 13,50 & 10,00 & 135,00 & 20,00 & 270,00 & 25,00 & 337,50 \\
\hline Aplic. de defensivos & $\mathrm{d} / \mathrm{h}$ & 9,00 & 8,00 & 72,00 & 16,00 & 144,00 & 20,00 & 180,00 \\
\hline Colheita & $\mathrm{d} / \mathrm{h}$ & & & & & & 0,90 & 76,50 \\
\hline Colheita & $\mathrm{h} / \mathrm{tr}$ & & & & & & 0,80 & 28,00 \\
\hline Despesas de colheita & $\mathrm{R} \$ / \mathrm{ha}$ & & & & & & & 35,00 \\
\hline Mãos-de-obra diversas & $\mathrm{d} / \mathrm{h}$ & 9,00 & 10,00 & 90,00 & 12,00 & 108,00 & 12,00 & 108,00 \\
\hline Outros serviços & $\mathrm{h} / \mathrm{tr}$ & 13,50 & 4,00 & 54,00 & 8,00 & 108,00 & 10,00 & 135,00 \\
\hline Serviços com veículos & $\mathrm{h} / \mathrm{tr}$ & 12,00 & 2,00 & 24,00 & 3,00 & 36,00 & 5,00 & 60,00 \\
\hline Despesas administr. & $\mathrm{R} \$ / \mathrm{ha}$ & & & 130,00 & & 230,00 & & 330,00 \\
\hline Sub-total & & & & 802,00 & & $1.332,50$ & & $1.834,50$ \\
\hline Total & & & & $1.443,60$ & & $2.517,10$ & & $3.506,60$ \\
\hline
\end{tabular}


mar adulto se reduz para $\mathrm{R} \$ 4.595,88$ para o caso de a densidade de plantio ser ampliada para 3.378 plantas/ha. Isto se deve à maior produtividade obtida nos serviços manuais.

No mesmo período de existência do pomar, a maior densidade apresenta maior produção acumulada e maior desembolso acumulado de recursos financeiros (Tabela 4).

A rentabilidade, medida pela TIR, se encontra entre $1,61 \%$ ao mês na densidade de 1.000 plantas/ha, e $1,71 \%$ ao mês na densidade de 3.378 plantas/ha. A rentabilidade, ao se investir em pomar de macieira, nas densidades estudadas, supera a rentabilidade que pode ser obtida em empreendimentos florestais na região, a qual se aproxima de $1 \%$ ao mês (Kreuz \& Baú, 2001).

A variação da rentabilidade foi pequena entre as duas densidades testadas. Assim, pode-se recomendar o uso da densidade de 1.000 plantas/ha para o caso estudado, uma vez que isto implica menor necessidade de capital. Contudo, para as situações onde o capital não for escasso, em condições similares à estudada, pode-se recomendar o aumento da densidade de plantio, uma vez que isto implicará em uma boa rentabilidade para o recurso, o que está de acordo com Ferree et al. (1989). Jackson et al. (1986), Ferree et al. (1989) e Dejong et al. (1999) também não conseguiram ganhos expressivos de rentabilidade com o aumento da densidade.

Preocupação adicional diz respeito ao risco, principalmente no que se refere ao risco climático. O presente estudo não levou em consideração a possibilidade de granizo (ou geadas tardias), cuja ocorrência é freqüente na região produtora de Fraiburgo. Dado que as perdas por problemas climáticos são maiores em pomares em alta densidade (Ferree et al., 1989), este poderia ser mais um argumento (associado ao da rentabilidade) para, na em-

Tabela 3. Custo de produção de 1 ha de macieira cultivar Gala em idade adulta, na densidade de 1.000 plantas/ha, em outubro de 2000 .

\begin{tabular}{|c|c|c|c|c|}
\hline Descrição & Unidade & $\begin{array}{c}\text { Preço } \\
\text { (R\$/unid.) }\end{array}$ & $\begin{array}{l}\text { Quantidade } \\
\text { (unid./ha) }\end{array}$ & $\begin{array}{l}\text { Valor } \\
\mathrm{R} \$ / \text { ha }\end{array}$ \\
\hline \multicolumn{5}{|l|}{ Insumos } \\
\hline Fertilizantes & & & & 369,36 \\
\hline Fungicidas & & & & $1.205,02$ \\
\hline Inseticidas & & & & 478,07 \\
\hline Herbicidas & & & & 58,18 \\
\hline Sub-total & & & & $2.110,63$ \\
\hline \multicolumn{5}{|l|}{ Serviços } \\
\hline Aplicação de fertilizantes & $\mathrm{h} / \mathrm{tr}$ & 8,00 & 4 & 32,00 \\
\hline Aplicação de fertilizantes & $\mathrm{d} / \mathrm{h}$ & 9,00 & 6 & 54,00 \\
\hline Podas & $\mathrm{d} / \mathrm{h}$ & 9,00 & 18 & 162,00 \\
\hline Conduções diversas & $\mathrm{d} / \mathrm{h}$ & 9,00 & 10 & 90,00 \\
\hline Raleio & $\mathrm{d} / \mathrm{h}$ & 9,00 & 30 & 270,00 \\
\hline Arqueamento & $\mathrm{d} / \mathrm{h}$ & 9,00 & 25 & 225,00 \\
\hline Aplicação de defensivos & $\mathrm{h} / \mathrm{tr}$ & 8,00 & 30 & 240,00 \\
\hline Mãos-de-obra diversas & $\mathrm{d} / \mathrm{h}$ & 9,00 & 25 & 225,00 \\
\hline Serviços diversos de máquinas & $\mathrm{h} / \mathrm{tr}$ & 8,00 & 5 & 40,00 \\
\hline Outras despesas & & & & 200,00 \\
\hline Sub-total & - & - & - & $1.538,00$ \\
\hline \multicolumn{5}{|l|}{ Colheita } \\
\hline Mão-de-obra & $\mathrm{d} / \mathrm{h}$ & 9,00 & 85 & 765,00 \\
\hline Serviços de máquinas & $\mathrm{h} / \mathrm{tr}$ & 8,00 & 35 & 280,00 \\
\hline Outras despesas & & & & 350,00 \\
\hline Sub-total & - & - & - & $1.395,00$ \\
\hline Custo/ha & - & - & - & $5.043,63$ \\
\hline
\end{tabular}


Tabela 4. Produtividade, desembolso, receitas e fluxo de caixa em duas densidades de plantio de macieira durante a vida do pomar, em outubro de 2000.

\begin{tabular}{|c|c|c|c|c|c|c|c|c|}
\hline \multirow[t]{2}{*}{ Ano } & \multicolumn{2}{|c|}{$\begin{array}{l}\text { Produtividade } \\
\text { (t/ha) }\end{array}$} & \multicolumn{2}{|c|}{$\begin{array}{c}\text { Desembolso } \\
\text { (R\$/ha) }\end{array}$} & \multicolumn{2}{|c|}{$\begin{array}{l}\text { Receitas } \\
(\mathrm{R} \$ / \mathrm{ha})\end{array}$} & \multicolumn{2}{|c|}{$\begin{array}{l}\text { Fluxo de caixa } \\
\text { (R\$/ha) }\end{array}$} \\
\hline & $1.000 \mathrm{pl} . / \mathrm{ha}$ & $3.378 \mathrm{pl} . / \mathrm{ha}$ & $1.000 \mathrm{pl} . / \mathrm{ha}$ & $3.378 \mathrm{pl} . / \mathrm{ha}$ & $1.000 \mathrm{pl} . / \mathrm{ha}$ & $3.378 \mathrm{pl} . / \mathrm{ha}$ & $1.000 \mathrm{pl} . / \mathrm{ha}$ & $3.378 \mathrm{pl} . / \mathrm{ha}$ \\
\hline 0 & 0 & 0 & $6.423,00$ & $12.203,40$ & 0,00 & 0,00 & $-6.423,00$ & $-12.203,40$ \\
\hline 1 & 0 & 0 & $1.443,60$ & $1.443,60$ & 0,00 & 0,00 & $-1.443,60$ & $-1.443,60$ \\
\hline 2 & 0 & 5 & $2.517,10$ & $2.656,60$ & 0,00 & $1.181,25$ & $-2.517,10$ & $-1.475,35$ \\
\hline 3 & 5 & 20 & $3.506,60$ & $3.925,10$ & $1.125,00$ & $4.725,00$ & $-2.381,60$ & 799,90 \\
\hline 4 & 20 & 30 & $4.206,63$ & $4.037,88$ & $4.500,00$ & $7.087,50$ & 293,37 & $3.049,62$ \\
\hline 5 & 30 & 45 & $4.485,63$ & $4.456,38$ & $6.750,00$ & $10.631,25$ & $2.264,37$ & $6.174,87$ \\
\hline 6 & 45 & 50 & $4.904,13$ & $4.595,88$ & $10.125,00$ & $11.812,50$ & $5.220,87$ & $7.216,62$ \\
\hline 7 & 50 & 50 & $5.043,63$ & $4.595,88$ & $11.250,00$ & $11.812,50$ & $6.206,37$ & $7.216,62$ \\
\hline 8 & 50 & 50 & $5.043,63$ & $4.595,88$ & $11.250,00$ & $11.812,50$ & $6.206,37$ & $7.216,62$ \\
\hline 9 & 50 & 50 & $5.043,63$ & $4.595,88$ & $11.250,00$ & $11.812,50$ & $6.206,37$ & $7.216,62$ \\
\hline 10 & 50 & 50 & $5.043,63$ & $4.595,88$ & $11.250,00$ & $11.812,50$ & $6.206,37$ & $7.216,62$ \\
\hline 11 & 50 & 50 & $5.043,63$ & $4.595,88$ & $11.250,00$ & $11.812,50$ & $6.206,37$ & $7.216,62$ \\
\hline 12 & 50 & 50 & $5.043,63$ & $4.595,88$ & $11.250,00$ & $11.812,50$ & $6.206,37$ & $7.216,62$ \\
\hline 13 & 50 & 50 & $5.043,63$ & $4.595,88$ & $11.250,00$ & $11.812,50$ & $6.206,37$ & $7.216,62$ \\
\hline 14 & 50 & 50 & $5.043,63$ & $4.595,88$ & $11.250,00$ & $11.812,50$ & $6.206,37$ & $7.216,62$ \\
\hline 15 & 50 & 50 & $5.043,63$ & $4.595,88$ & $11.250,00$ & $11.812,50$ & $6.206,37$ & $7.216,62$ \\
\hline 16 & 50 & 50 & $5.043,63$ & $4.595,88$ & $12.750,00$ & $11.812,50$ & $7.706,37$ & $7.216,62$ \\
\hline Total & 600 & 650 & $77.922,99$ & $79.277,64$ & $136.500,00$ & $153.562,50$ & $58.577,01$ & $74.284,86$ \\
\hline
\end{tabular}

presa estudada, optar-se pela opção de menor densidade de plantio.

O custo do quilo de maçã produzido também não diferiu de forma expressiva entre as densidades, variando de $\mathrm{R} \$ 0,147 / \mathrm{kg}$ na densidade de 1.000 plantas/ha a R \$ 0,142/kg na densidade de 3.378 plantas/ha. Pelo fato de o preço esperado de venda estar próximo a $\mathrm{R} \$ 0,225 / \mathrm{kg}$, tem-se uma margem de $54 \%$ $(0,225 / 0,147)$ em cada quilo a ser produzido e comercializado na menor densidade de plantio, a qual pode ser considerada boa. Na situação de maior densidade de plantio, a margem é superior, atingindo $66 \%(0,236 / 0,142)$. À medida que o preço cresce do seu custo de produção até o preço de mercado, a decisão sobre qual densidade de plantio adotar não se altera. Dejong et al. (1997) encontrou vantagens econômicas em relação às maiores densidades do pessegueiro à medida que o preço da fruta cresceu.

\section{Conclusões}

1. A taxa de retorno de investimentos na cultura da macieira, a qual varia em torno de 1,61 e 1,71\% ao mês, independentemente de a densidade de plantio ser de 1.000 ou 3.378 plantas/ha, é considerada boa.
2. O uso de 1.000 plantas/ha propicia uma boa taxa de retorno de investimento (1,61\% ao mês) sendo, portanto, recomendada.

3. O custo do quilo de maçã produzido não varia à medida que se aumenta a densidade de plantio de 1.000 para 3.378 plantas/ha.

\section{Referências}

BARRIT, H. B. Apple orchard management. In: ENCONTRO NACIONAL SOBRE FRUTICULTURA DE CLIMA TEMPERADO, 2., 1999, Fraiburgo. Anais... Caçador: Epagri, 1999. p. 1-8.

CAHN-SHAHARUDIN, M. Small apple trees and closer spacing: the Duth experience is it for us? Orchardist of New Zealand, Wellington, v. 64, n. 1, p. 11-13, 1991.

DEJONG T. M.; TSUIJI, W.; DOYLE, J. F.; GROSSMAN, Y. L. Comparative economic efficiency of four peach production systems in California. HortScience, Alexandria, v. 34, n. 1, p. 73-78, 1999.

DEJONG T. M.; TSUIJI, W.; DOYLE, J. F.; GROSSMAN, Y. L. L. Do high density systems really pay? Evaluation of high density systems for cling peaches. Acta Horticulturae, Leuven, n. 451, p. 599-604, 1997. 
EMPRESA DE PESQUISAAGROPECUÁRIADE SANTA CATARINA (Florianópolis, SC). Sistema de produção para a cultura da macieira: Santa Catarina. 3. ed. rev. Florianópolis, 1991. 71 p. (Empasc/Acaresc. Sistemas de Produção, 19).

FERREE, D. C.; FUNT, R. C.; BISHOP, B. L. Yield and production efficiency of four apple cultivars in selected orchard management systems. Journal of the American Society for Horticultural Science, Alexandria, v. 114, n. 6 , p. 863-868, 1989.

GOEDEGEBURE, J. Economic aspects of high density plantings in apple-growing in the Netherlands. Acta Horticulturae, Leuven, n. 114, p. 389-397, 1980.

JACKSON, J. E.; WHITE, G. C.; DUNCAN, C. Economic appraisal of orchards of Cox's orange pippin apple on M. 9 and MM.106 rootstocks. Acta Horticulturae, Leuven, n. 160, p. $383-387,1986$.

KREUZ, C. L. Custo de produção de maçã. Pesquisa Agropecuária Brasileira, Brasília, v. 27, n. 5, p. 721-726, maio 1992.

KREUZ, C. L.; BAÚ, N. Análise da rentabilidade do cultivo de Pinus taeda na região de Caçador, SC. Agropecuária Catarinense, Florianópolis, v. 15, n. 2, p. 8-10, 2001.

KREUZ, C. L.; PETRI, J. L.; MONDARDO, M. Capacidade produtiva de macieiras cv. Fuji em diversos espaçamentos, com o uso de mudas livres de viroses. Pesquisa Agropecuária Brasileira, Brasília, v. 31, n. 2, p. 121-127, fev. 1996.

PALMER, J. W.; ADAMS, H. M. Early results with intensive systems of apples on virus-free M. 9 rootstock in New Zealand. Acta Horticulturae, Leuven, n. 451, p. 487-493, 1997.

PETRI, J. L. Fatores edafoclimáticos. In: EMPRESA DE PESQUISAAGROPECUÁRIA DE SANTA CATARINA (Florianópolis, SC). Manual da cultura da macieira. Florianópolis, 1986. p. 50-58.
PETRI, J. L.; KREUZ, C. L.; RAASCH, Z. Influência da densidade de plantio na coloração dos frutos e na produção da macieira cv. Fuji nas duas primeiras produções. Revista Brasileira de Fruticultura, Cruz das Almas, v. 13, n. 1, p. 25-34, 1991.

ROBINSON, T. L.; LAKSO, A. N. Bases of yield and production efficiency in apple orchard systems. Journal of the American Society for Horticultural Science, Alexandria, v. 116, n. 2, p. 188-194, 1991.

ROBINSON, T. L.; HOYING, S. A.; SMITH, W. H. Apple orchard systems trials in New York. Compact Fruit Tree, Wenatchee, v. 29, p. 107-113, 1996.

ROBINSON, T. L.; LAKSO, A. N.; CARPENTER, S. G. Canopy development, yield, and fruit quality of 'Empire' and 'Delicious'apple trees grown in four orchard production systems for ten years. Journal of the American Society for Horticultural Science, Alexandria, v. 116, n. 2, p. 179-187, 1991a.

ROBINSON, T. L.; LAKSO, A. N.; REN, Z. Modifying apple tree canopies for improved production efficiency. HortScience, Alexandria, v. 26, n. 8, p. 1005-1012, 1991 b.

SANSAVINI, S. Nuovi impianti e qualità delle mele. In: SANSAVINI, S.; ERRANI, A. Frutticoltura ad alta densità: impianti, forme di allevamento, e tecniche di potatura. Bologna: Edagricole, 1998. p. 3-25.

VIEIRA SOBRINHO, J. D. Matemática financeira. São Paulo: Atlas, 1997. 409 p.

WERTHEIM, S. J.; JÄGER, A. de; DUYZENS, M. J. J. P. Comparison of single row and multi-row planting systems with apple, with regard to productivity, fruit size and color, and light. Acta Horticulturae, Leuven, n. 160, p. 243-258, 1986.

WINTER, F. Modeling the biological and economic development of an apple orchard. Acta Horticulturae, Leuven, n. 160, p. 353-360, 1986. 